\title{
Metals and RNA: scenes from a road trip
}

\author{
VICTORIA J. DEROSE \\ Department of Chemistry and Biochemistry, University of Oregon, Eugene, Oregon 97403-1253, USA
}

About twenty years ago, I began working with ribozymes as an Assistant Professor trained in the world of metalloproteins. In retrospect, moving to the RNA world was a naïve but brilliant move. Naïve, because I had little idea of the complexities and challenges inherent to ribozyme studies. Brilliant, for personal career satisfaction, because I've been present for amazing discoveries in RNA biology over two decades.

In establishing independent careers, Assistant Professors in Chemistry are expected to make a distinct shift in areas. In 1995, I had graduate and postdoctoral training in structurefunction of protein metalloenzymes; rather than switching protein targets, I switched biomolecules. Ribozymes were in discovery, were fascinating, and needed $\mathrm{Mg}^{2+}$ for function. Naïvely simple questions were unanswered: How many metals contributed to activity? How were they captured, what were their RNA binding sites and affinities? How similar were ribozymes, in active-site structure and mechanism, to protein-based polymerases and nucleases? How in heaven's name could RNA, with its seemingly limited structural diversity but a forest of phosphate anions, specifically poise cationic metal ions for targeted catalysis? The Hammerhead Ribozyme (HHRz), discovered by Bruening and as constructed in Uhlenbeck's laboratory for pioneering biochemical studies, was small enough to handle for structure investigations. Indeed a first X-ray crystal structure had been reported by McKay's laboratory in 1994. Pyle had recently written about "ribozymes, a distinct class of metalloenzymes." To me, this presented a viable bioinorganic field.

As a spectroscopist, I thought that we could contribute through direct observation of the metal environment via surrogate $\mathrm{Mn}$ (II) substitutions and metal-based spectroscopies. These methods were de rigeur for the field of metalloproteins, and it seemed straightforward to propose applying them to ribozymes. In the metalloprotein field, trapping reaction intermediates and monitoring their structures was routine, if not trivial. How difficult could it be to apply these powerful methods to a little piece of catalytic RNA?

In the mid-1990s, the HHRz, as the smallest naturally-occurring ribozyme performing the simplest nucleolytic bond

\footnotetext{
Corresponding author: derose@uoregon.edu

Article and publication date are at http://www.rnajournal.org/cgi/doi/10. 1261/rna.050666.115. Freely available online through the RNA Open Access option.
}

cleavage reaction, appeared to be a great candidate for the Rosetta stone of RNA catalysis. The HHRz had appealing properties: a fascinating (to me) source in plant viroids; a deconstructed model system of two strands short enough for solid-phase synthesis methods and thus site-specific substitutions; a crystal structure that was the first new RNA structure since those of tRNAs. Founding mechanistic studies were performed by several investigators, and a rich substitutionfunction library was being formed. These early studies were enabled by the small companies (and with every Dharmacon invoice, I wished I had bought stock) that grew out of the recognition that ribozymes could target and cleave any nucleic acid sequence and thus be potential therapeutics. (A bonus highlight of being in this field has been watching the fascinating, rapid evolution of this technology from ribozymes to siRNA to CRISPR, with basic discoveries moving hand-in-hand with biotechnology. RNA and the Society should take credit for creating a community supporting such trajectories.)

We envisioned that a ribozyme functional metal site could be discretely populated and examined, but in retrospect that simple assumption imported from the metalloprotein field was not obviously applied to RNA. In a cellular environment of $\sim \mathrm{mM} \mathrm{Mg}^{2+}$, why would ribozymes need the active-site metal ion to be particularly high-affinity? "A sea of metals" or "needle in a haystack" were standard terms applied to finding a ribozyme active-site metal ion. Ribozymes also require cations for folding, and so for metal-dependent activity analyses there was a challenge of separating requirements for folding and activity. The HHRz constructs in use had a relatively high cation requirement for activity that did not match physiological environments. There was also a pesky counterion atmosphere to consider. Still, applying electron paramagnetic resonance (EPR) methods in basic binding studies, we were very interested to find that the HHRz had an apparently high-affinity metal site. Through "advanced EPR techniques" we elucidated the direct ligands to $\mathrm{Mn}$ (II) in this site, counted bound waters, and identified the bound guanine through direct ${ }^{15} \mathrm{~N}$ labeling. These data mapped onto density observed in McKay's early crystal structure, the "A9/G10.1" site that was related to function through mutagenesis in other studies.

(C) 2015 DeRose This article, published in $R N A$, is available under a Creative Commons License (Attribution-NonCommercial 4.0 International), as described at http://creativecommons.org/licenses/by-nc/4.0/. 
To us, the most interesting aspect was that in solution conditions, this RNA site was unambiguously selectively populated at low $\mathrm{Mn}$ (II) concentrations, supporting the instinct that a functional ribozyme metal ion site was also high-affinity.

Following McKay's early structure, continuous high-impact work from Bill Scott's laboratory provided HHRz crystallographic structures of various sequences, substitutions, and ionic conditions. Each of these show subtle variations in local arrangements around the HHRz active site, illustrating a common theme that ribozyme active sites are plastic and very sensitive to small changes. In particular, the fact that ribozymes partake in substrate-assisted catalysis means that employing substrate inhibition to trap inactive models can be problematic. As in the metalloenzyme field, mechanistic studies are a prerequisite to meaningful interpretation of structure, and in concert, structures inspire new mechanistic tests. An early inconsistency between structure and mechanistic work created a puzzle for the HHRz that is still unsolved. From results of phosphorothioate metal-rescue studies, Herschlag and coworkers predicted an active-site metal that bridged both the A9 phosphate and the cleavage site. Early $\mathrm{HHRz}$ structures showed those ligands too far apart to match this expectation. Several other inhibitory substitutions could not be explained based on structures of the original $\mathrm{HHRz}$ constructs. Some explanation came following a major turning point when, in an effort to use $\mathrm{HHRz}$ constructs to control gene expression, Khvorova and coworkers at Amgen searched biology for optimized sequences. In 2003 they reported extensions to the HHRz stems that were predicted to interact based on covariance and models. Including these extended loops increased $\mathrm{HHRz}$ activity at decreased metal ion concentrations. Ensuing results from crystallography by Scott and coworkers, FRET by Pardi and coworkers, and our spin-label EPR studies, all agreed that these tertiary interactions in "extended, native" $\mathrm{HHRz}$ sequences resulted in a more compact active site. The importance of including tertiary interactions in $\mathrm{HHRz}$ sequences was a critical early demonstration of powerful bioinformatics tools for predicting RNA structure motifs, as well as a warning against the "snip too far," in the words of Uhlenbeck, when developing in vitro models.

Another discovery that gained much attention was the fact that the HHRz, in its shortened context, and other "small" ribozymes such as the hairpin, exhibit significant activity in molar concentrations of monovalent cations. Reported by Scott in relation to activity within $\mathrm{HHRz}$ crystals, and further examined by Herschlag and others, this ultimately was important in identifying and highlighting the full suite of contributions to ribozyme catalysis besides divalent cations. In particular, Bevilacqua and coworkers had discovered nucleobase-supported acid-base catalysis in the Hepatitis Delta Virus ribozyme, and established a framework for interpreting $\mathrm{pH}$-rate profiles needed for such studies. Applied to the $\mathrm{HHRz}$, and in concert with structural information, a role for conserved nucleobase G12 as general base has been pro- posed. At the same time, Pardi and coworkers established a distinct metal-ion dependence on HHRz rates that correlates with Lewis acid properties of the metals. Similarly, our group repeated phosphorothioate substitution studies in context of the "native, extended" $\mathrm{HHRz}$ that support intimate involvement of a metal in catalysis. The current model for HHRz activity therefore includes both metals and nucleobases, but in my opinion the details are not yet set. I think the field would be remiss without supporting the continued, albeit challenging, dedicated efforts needed to create textbook-ready mechanistic models for this and other ribozymes.

Two areas regarding ribozymes and metal-RNA interactions in general seem obvious directions for the next decade(s). Known ribozyme activity is scattered in biology, but bioinformatics approaches locate putative ribozyme sequences in all branches of life. Self-cleaving sequences may be evolutionarily conserved for as-yet unknown function. Alternatively or in addition, these sequences may be tolerated if they self-destruct once transcribed. A growing toolbox of clever high-throughput genomic and structure/function methods provides powerful approaches to address these and other questions about noncoding RNAs. A more general topic concerns the evolution of RNA-metal interactions. Either preserving or avoiding specific metal-RNA interactions seems an important factor, given their power to dramatically alter structure or install activity. As the earth's ancient metallochemistry changed dramatically, RNA-metal interactions would have to respond. I think demonstrations of extant ribosome and other RNA reactivity with reduced Fe (Williams, Hud, and coworkers) are provocative in this regard. Also very interesting are new discoveries of metal-dependent riboswitches reported by Winkler, Breaker, and Storz to regulate metal ion uptake. The evolution of specific metal-RNA regulatory elements makes sense, and these riboswitches seem to be selective for different ions including $\mathrm{Mn}, \mathrm{Co}$, and $\mathrm{Ni}$. Understanding properties allowing RNA to selectively recognize these ions is a fascinating challenge.

Our own current research has evolved to looking at different aspects of metal-RNA interactions. We are exploring how cellular RNA is acting as a target or sink for metal-based compounds such as the platinum-based pharmaceuticals, and in using those compounds as crosslinking agents for high-throughput RNA structure analysis. Analytical and biochemical approaches are showing a surprising amount of interaction between these drugs and the ribosome as well as tRNA, and tools are in place to locate other RNA sites along with plans to track the implications of these interactions. Such interactions were broadly detected about twenty years ago, but were dismissed by researchers at the time because RNA was considered a transient molecule. Twenty years ago, the vast level of RNA regulation underlying gene expression, along with RNA surveillance mechanisms and damage responses, were unknown and possibly unimagined. It's difficult to think of a faster-moving field than the RNA world, and I'm grateful to have jumped into it. 

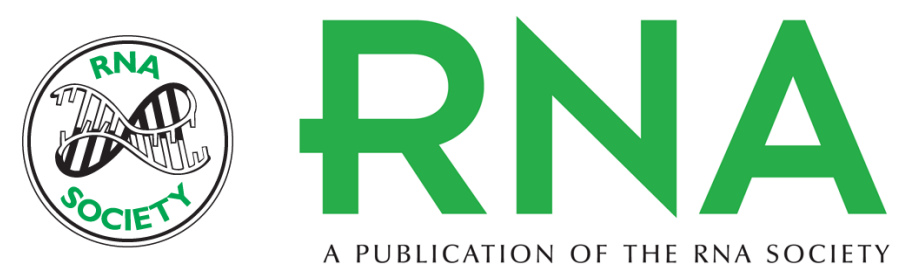

A PUBLICATION OF THE RNA SOCIETY

\title{
Metals and RNA: scenes from a road trip
}

\author{
Victoria J. DeRose
}

RNA 2015 21: 595-596
Open Access Freely available online through the RNA Open Access option.
Creative This article, published in $R N A$, is available under a Creative Commons License Commons (Attribution-NonCommercial 4.0 International), as described at License http://creativecommons.org/licenses/by-nc/4.0/. Email Alerting
Service
top right corner of the article or click here.

To subscribe to RNA go to:

http://rnajournal.cshlp.org/subscriptions

C 2015 DeRose; Published by Cold Spring Harbor Laboratory Press for the RNA Society 